\title{
Retrospective study assessing efficacy and safety of left atrial appendage occlusion
}

\author{
Misikova $\mathrm{S}^{1}$, Haramiova $\mathrm{Z}^{2,3}$, Juhas $\mathrm{S}^{1}$, Ondusova $\mathrm{D}^{1}$, Kobliskova $\mathrm{Z}^{2}$, Zajac $\mathrm{I}^{3}$, Tesar $\mathrm{T}^{2}$ \\ East Slovak Institute of Cardiovascular Disease, Arrhythmology Department of Cardiologic Clinic, Kosice, \\ Slovakia.tesar@fpharm.uniba.sk
}

\section{ABSTRACT}

OBJECTIVE: To describe the initial experience with Amplatzer Amulet in left atrial appendage occlusion in Slovakia. To evaluate procedural efficacy and safety.

BACKGROUND: Atrial fibrillation increases the risk of stroke. While anticoagulation therapy can reduce the risk of stroke, it is associated with bleeding risk and often unsatisfactory prescribed. Most thrombi form in the left atrium appendage, hence left atrium appendage occlusion may be a suitable therapeutic alternative for these patients.

METHODS: This is an observational, retrospective, single-centre, case-series study including 30 patients with atrial fibrillation at a high risk of stroke, undergoing left atrial appendage occlusion from June 2015 to December 2018.

RESULTS: The left atrial appendage was successfully closed in 29 (96.7 \%) patients. Three months after the procedure, 4 patients had small leaks $(<2 \mathrm{~mm})$. No complications were reported so far. Prior to the procedure, patients mostly received low molecular weight heparin $(53.3 \%)$. Three months after the procedure, patients mostly received acetylsalicylic acid (60.7 \%) and clopidogrel (32.1\%).

CONCLUSION: Left atrial appendage occlusion was shown to be an effective and safe alternative to anticoagulation therapy in patients with atrial fibrillation, at a high risk of stroke and bleeding. The procedure is safe, when performed carefully even by less experienced cardiologists (Tab. 6, Fig. 4, Ref. 29). Text in PDF www.elis.sk

KEY WORDS: atrial fibrillation, stroke, haemorrhage, left atrial appendage occlusion, Amplatzer Amulet.

Abbreviations: ASA - acetylsalicylic acid, ACP - Amplatzer Cardiac Plug, ANOVA - analysis of variance, AFib - atrial fibrillation, DOAC - direct oral anticoagulants, ICE - intracardiac echocardiography, LAA - left atrial appendage, LAAO - left atrial appendage occlusion, LWMH - low molecular weight heparin, RAO - right anterior oblique, TEE - transesophageal echocardiography

\section{Introduction}

To date, no other rhythm disorder has attracted as much attention as atrial fibrillation (AFib). It is associated with an increased morbidity and mortality, and its treatment is financially demanding. Furthermore, AFib is not merely a simple heart rhythm disorder; it has also been shown to increase the risk of thromboembolic complications. Stroke, regardless of its form (paroxysmal, persistent, or permanent), is one of the most severe thromboembolic

${ }^{1}$ East Slovak Institute of Cardiovascular Disease, Arrhythmology Department of Cardiologic Clinic, Kosice, Slovakia, ${ }^{2}$ Department of Organisation and Management of Pharmacy, Comenius University, Bratislava, Slovakia, and ${ }^{3} \mathrm{mHELZ}$, non-profit organization, Bratislava, Slovakia

Address for correspondence: T. Tesar, PharmDr, PhD, MPH, MBA, Department of Organisation and Management of Pharmacy, Comenius University, Bratislava, Ulica odbojarov 10, SK-832 32 Bratislava 3, Slovakia.

Acknowledgement: The study was supported by mHELZ, o.z., a nonprofit patient organization. disorders associated with significant health risks (1). To evaluate the stroke-risk in AFib patients, the CHA2DS2VASc scoring system was developed (2). However, bleeding is the major adverse effect of anticoagulation therapy. The risk of bleeding among patients receiving anticoagulation therapy can be evaluated with the HAS-BLED scoring system (3). The considerable overlap of the risk factors used by both the CHA2DS2VASc and HAS-BLED scoring systems stresses the narrow risk-benefit threshold of anticoagulation therapy in AFib patients, which is also reflected in clinical practice.

Clinically significant emboli originate in the left atrial appendage (LAA) in $91 \%$ and $57 \%$ of cases of patients with non-valvular and valvular AFib, respectively (4). Therefore, endocardial left atrial appendage occlusion (LAAO) may be an appropriate alternative to anticoagulation therapy in high-risk patients, as it significantly decreases the risk of stroke without simultaneously increasing the risk of bleeding. Catheter-based LAAO has been developing since the turn of the millennium (5). Several types of catheter occluders are available, including Watchman (Boston Scientific) and the second generation of the Amplatzer Cardiac Plug (ACP) Amulet (St. Jude Medical), which are the most frequently utilized in clinical practice.

According to the latest Guidelines of the European Society of Cardiology for the management of AFib from 2016, percutaneous LAAO may be considered in patients at high risk of stroke 
609-618

with a concurrent contraindication to long-term anticoagulant treatment (1).

To date, no Slovak national data on the effectiveness and safety of the LAAO procedure have been reported. The current paper reports on the initial experience with the ACP Amulet device in LAAO at an inpatient hospital setting in one of the leading institutes for cardiovascular diseases in Slovakia. This retrospective study was designed to assess the indication, effectiveness and safety of the LAAO procedure and the incidence of complications during follow-up in Slovakia at this centre.

\section{Materials and methods}

\section{Study population and setting}

This study analyses data from 30 AFib patients, who underwent LAAO from June 2015 to December 2018 at our institute. In one patient, LAAO was successfully performed twice to close the LAA. All the patients were thoroughly informed about the procedure including possible complications and they provided their written informed consent prior to the procedure.

LAAO was indicated if patients met the following inclusion criteria:

1) contraindication of long-term anticoagulation treatment; and

2) $\mathrm{CHA}_{2} \mathrm{DS}_{2}$ VASc score $\geq 2$.

For the pre-procedural imaging of LAA, all the patients underwent transoesophageal echocardiography (TEE) to evaluate: the morphology and size of the LAA, and presence of adjacent lobes and thrombi in the LAA. Patients were not indicated for the LAAO if one the following conditions was confirmed: 1) adjacent lobes in the landing zone; or 2) depth of the LAA $<11 \mathrm{~mm}$. We did not use CT examination.

In cases, where LAAO indication was disputable, a consensual decision was made by 3 cardiologists specializing in arrhythmology, structural interventions and echocardiography. The LAAO procedure was not indicated in patients, who met at least one of the following exclusion criteria:

1. presence of thrombi in LAA;

2. any inflammatory disease;

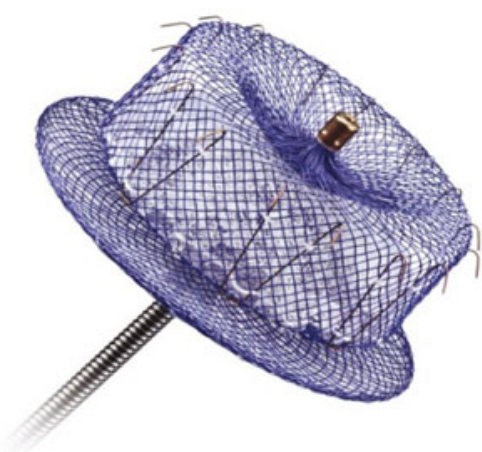

Fig. 1. The second generation of Amplatzer Cardiac plug - Amulet (St. Jude Medical).

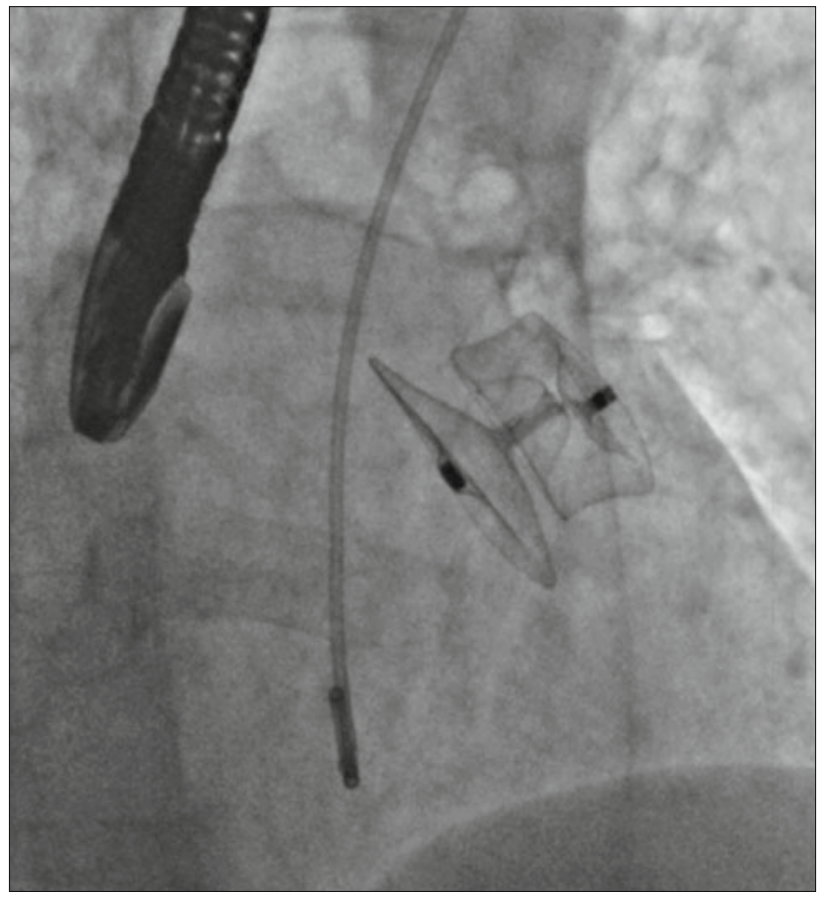

Fig. 2. Fluoroscopic display of final occluder placement of the Amplatzer Cardiac plug - Amulet (St. Jude Medical) placement; projection: right anterior oblique, RAO $30^{\circ}$, cranial $20^{\circ}$.

3. any concomitant disease preventing full heparinization during the procedure;

4. severe heart failure;

5. myocardial infarction or stroke within the past 3 months; or

6. overall poor health with expected survival of less than one year. Based on the present contraindication to anticoagulation therapy, we divided the patients in our analysis sample into four groups: Group 1: patients with a medical history of haemorrhagic stroke or severe bleeding;

Group 2: patients with recurrent bleeding despite an adequate anticoagulation therapy;

Group 3: patients with a medical history of ischemic stroke despite an adequate anticoagulation therapy (after excluding all other possible causes of stroke); and

Group 4: patients with other reasons for high bleeding risk.

\section{Materials}

All the patients in the study sample underwent the LAAO procedure with the second-generation ACP Amulet (St. Jude Medical) (Fig. 1). TEE examination was performed on the Philips iE33 device using real-time 3D and X-plane modality.

\section{Procedure}

In 29 out of 30 patients, the LAAO procedure was guided by TEE and fluoroscopic imaging. The esophageal structure prevented the introduction of the TEE probe in one patient; as the result, intracardiac echocardiography imaging in local anaesthesia was used to guide LAAO. 
The ACP Amulet device was implanted into the LAA via the right femoral vein by transseptal puncture using a delivery sheath. The transseptal puncture was performed in the infero-posterior part of the LAA under TEE guidance with a real-time 3D control. Afterwards, the LAA was probed with a pigtail catheter guided by TEE and fluoroscopy in two right $30^{\circ}$ oblique projections (right anterior oblique [RAO]): caudal and cranial at $10-20^{\circ}$ to obtain the precise landing zone dimensions.

Occluder size was selected according to the manufacturer's sizing chart based on the diameter of the LAA landing zone dimensions (diameter and depth). The landing zone was precisely measured at a depth of 10-11 mm from the LAA orifice with TEE and fluoroscopy in both projections stated earlier. The final occluder device was oversized by approximately $3-6 \mathrm{~mm}$ compared to the final measurement, as this was shown to improve its stability and the overall seal of the LAA ensuring low leak-rate.

If a satisfactory position of the occluder lobe meeting all the required criteria for correct placement was achieved, the procedure followed by unfolding the occluder disc. The stability and proper function of the LAA occlusion was controlled by TEE and contrast fluoroscopy. The occluder device position had to meet the following five criteria: 1) lobe compression 2) lobe and disc separation, 3) concave disc placement, 4) coaxial orientation, and 5) location of two thirds of the lobe distal to the circumflex coronary artery. The first 3 visible criteria were shown on Figure 2, displaying the

Tab. 1. Sociodemographic and clinical characteristics at baseline.

\begin{tabular}{lc}
\hline & $\begin{array}{c}\text { Sample size } \\
(\mathrm{n}=30)\end{array}$ \\
\hline Gender, n (\%) & $12(40.0)$ \\
Female & $18(60.0)$ \\
Male & \\
\hline Age, years & $71.3(7.5)$ \\
Mean (SD) & $71(65.5,77.8)$ \\
Median (Q1, Q3) & $57-84$ \\
Range: min-max & \\
Type of atrial fibrillation, n (\%) & $6(20.0)$ \\
Paroxysmal & $15(50.0)$ \\
Persistent & $9(30.0)$ \\
Permanent & \\
\hline Patient stroke risk status, CHA_DS,VASc score & $3.8(1.3)$ \\
Mean (SD) & $4(3,5)$ \\
Median (Q1, Q3) & $2-6$ \\
Range: min-max & \\
\hline Patient bleeding risk status, HASBLED score & $3.1(1)$ \\
Mean (SD) & $3(3,4)$ \\
Median (Q1, Q3) & $1-4$ \\
Range: min-max & \\
\hline Medical history prior to procedure, n (\%) & $28(93.3)$ \\
Arterial hypertension & $13(43.3)$ \\
Ischemic stroke & $12(40.0)$ \\
Diabetes mellitus & $11(36.7)$ \\
Ischemic heart disease & $5(16.7)$ \\
Haemorrhagic stroke & $5(16.7)$ \\
Chronic renal insufficiency & $3(10.0)$ \\
Atrial septum defect & $1(3.3)$ \\
Thrombophilic condition & $1(3.3)$ \\
Thrombus in left atrial appendage (resolved prior procedure) \\
SD - standard deviation, Q1 - first quartile, Q3 - third quartile \\
$\quad$
\end{tabular}

final occluder placement. If these criteria were met even after a five-minute mechanic pull (tug test), the occluder device was released from the delivery cable.

After the procedure, low-molecular-weight heparin and dual antiplatelet therapy were administered if the patients' bleeding risk allowed. Echocardiographic and X-ray controls were performed one day later. Patients were released to outpatient care, majority receiving a dual antiplatelet treatment.

During the first follow-up, which was scheduled 3 months after the LAAO procedure, the LAA closure was assessed with TEE for leaks and device thrombi. Additionally, the mitral valve function was evaluated. If the LAA closure was deemed optimal, patients continued with antiaggregating monotherapy unless their condition required a treatment change.

\section{Statistical analysis}

Based on the distribution of data, continuous data were summarized as the means, standard deviation, and minimum and maximum values, where applicable (for normal distribution) or median and 25 th and 75 th percentile (for skewed distribution). Categorical variables were summarized as frequencies and percentages. LAAO procedural success rates were calculated as percentages of the total number of patients undergoing the LAAO procedure. The Pearson's product moment correlation coefficient was used to assess linear associations between continuous variables (procedure times and operator experience). The relationship between CHA2DS2VASc and HASBLED scores in different LAAO indication groups was tested using a one-way analysis of variance (ANOVA) with a post hoc Gabriel test due to the different sizes of the indication groups. Results were considered statistically significant with a two-sided $\mathrm{p}<0.05$. All statistical data analyses were performed with SPSS 25.0 software (IBM Corp., Armonk, NY, USA).

\section{Results}

We analysed 30 patients, 12 (40.0\%) female and 18 (60.0\%) male, undergoing LAAO. One patient underwent the LAAO procedure twice to successfully place the occluder device. The mean patient age was $71.3 \pm 7.5$ years. All the patients were diagnosed with AFib: 6 (20.0\%), $15(50.0 \%)$ and 9 (30.0\%), with paroxysmal, persistent and a permanent AFib form, respectively. The mean $\mathrm{CHA}_{2} \mathrm{DS}_{2}$-VASc score was $3.8 \pm 1.3$ (range: $2-6$ ) and the median $\mathrm{CHA}_{2} \mathrm{DS}_{2}$ VASc score was 4.0. The mean HASBLED score was $3.1 \pm 1.0$ (range: $1-4$ ) and the median HASBLED score was 3.0 (Tab. 1).

\section{Periprocedural characteristics}

Table 2 provides an overview of all evaluated periprocedural parameters. The LAA was successfully closed during the first LAAO procedure in 28 out of 30 patients $(93.3 \%)$. Periprocedural complications preventing a successful LAA closure were observed in 1 patient only (3.3\%), who had overcome an ischemic stroke. The LAAO procedure with this patient was discontinued due to thrombi formation on the occluder device during 
Tab. 2. Periprocedural parameters.

\begin{tabular}{|c|c|}
\hline & $\begin{array}{l}\text { Sample size } \\
(\mathrm{n}=30)\end{array}$ \\
\hline \multicolumn{2}{|l|}{ Ejection fraction, \% } \\
\hline Mean (SD) & $53(7.2)$ \\
\hline Median (Q1, Q3) & $54(50,60)$ \\
\hline Range: $\min -\max$ & $35-65$ \\
\hline \multicolumn{2}{|l|}{ Mitral insufficiency, n (\%) } \\
\hline Absent & $7(23.3)$ \\
\hline First grade & $17(56.7)$ \\
\hline Second grade & $5(16.7)$ \\
\hline Third grade & $1(03.3)$ \\
\hline \multicolumn{2}{|c|}{ Left atrium diameter (short axis), mm } \\
\hline Mean (SD) & $45.3(4.9)$ \\
\hline Median (Q1, Q3) & $44.5(42,48)$ \\
\hline Range: $\min -\max$ & $37-56$ \\
\hline \multicolumn{2}{|c|}{ Left atrial appendage shape, $n$ (\%) } \\
\hline Cauliflower & $20(66.7)$ \\
\hline Windsock & $5(16.7)$ \\
\hline Cactus & $4(13.3)$ \\
\hline Chicken Wing & $1(3.3)$ \\
\hline \multicolumn{2}{|c|}{ Number of occluders per procedure, n (\%) } \\
\hline 1 & $27(90.0)$ \\
\hline 2 & $3(10.0)$ \\
\hline \multicolumn{2}{|c|}{ Success rate of left atrial appendage closure } \\
\hline During the first procedure & $28(93.3)$ \\
\hline During the second procedure & $1(3.3)$ \\
\hline Unsuccessful & $1(3.3)$ \\
\hline \multicolumn{2}{|l|}{ Total procedure time, min. } \\
\hline Mean (SD) & $104.5(30.7)$ \\
\hline Median (Q1, Q3) & $95(86.3,120)$ \\
\hline Range: $\min -\max$ & $50-190$ \\
\hline \multicolumn{2}{|l|}{ Fluoroscopic time, min. } \\
\hline Mean (SD) & $15.1(7.5)$ \\
\hline Median (Q1, Q3) & $11.8(9,19.1)$ \\
\hline Range: $\min -\max$ & $5.7-34.6$ \\
\hline \multicolumn{2}{|c|}{ Landing zone measurement with TEE, mm } \\
\hline Mean (SD) & $23.8(3.4)$ \\
\hline Median (Q1, Q3) & $24(22,25)$ \\
\hline Range: Min-Max & $17-32$ \\
\hline \multicolumn{2}{|c|}{ Landing zone measurement with fluoroscopy, mm } \\
\hline Mean (SD) & $24.6(4)$ \\
\hline Median (Q1, Q3) & $25(22.3,27.8)$ \\
\hline Range: $\min -\max$ & $16-33$ \\
\hline \multicolumn{2}{|l|}{ Occluder size, n (\%) } \\
\hline $28 \mathrm{~mm}$ & $12(40.0)$ \\
\hline $25 \mathrm{~mm}$ & $10(33.3)$ \\
\hline $34 \mathrm{~mm}$ & $4(13.3)$ \\
\hline $31 \mathrm{~mm}$ & $2(6.7)$ \\
\hline $20 \mathrm{~mm}$ & $1(3.3)$ \\
\hline $22 \mathrm{~mm}$ & $1(3.3)$ \\
\hline
\end{tabular}

placement; however the second LAAO procedure, which was carried out with high doses of heparin supplementation, was completed without complications. The LAAO procedure was unsuccessful in one patient with a Chicken Wing LAA with landing zone sizes of 20 and $25 \mathrm{~mm}$ measured by TEE and fluoroscopy, respectively. We used 2 occluder devices of 25 and $28 \mathrm{~mm}$, however, a tug test was performed in both instances and the occluder position was found to be unstable with a high risk of release. For safety reasons, we withdrew the occluder without closing the LAA. Additionally, intracardiac echocardiography (ICE) instead of TEE guidance was used with this patient during the LAAO procedure since the esophageal structure disabled the introduction of the TEE probe.

In 3 patients $(10.0 \%)$ a second occluder size was required due to the unstable positioning of the first occluder size. For 2 of these 3 patients, the LAAO procedure using the second occluder size was successful, while the LAAO procedure was unsuccessful with the third patient even with the second occluder size as described earlier.

In one patient, the delivery sheath was inserted via the left femoral vein instead of the right femoral vein due to obstruction of the venous system.

\section{Procedure time}

The average total LAAO procedure time was $104.5 \pm 30.7 \mathrm{~min}$ (range 50-190 $\mathrm{min}$ ) and the average fluoroscopy time was $15.1 \pm$ 7.5 min (range 5.7-34.6 min).

We found a negative correlation between the cardiologist's experience (assessed as days of experience performing the LAAO procedure) and the total procedural time $\mathrm{r}(28)=-0.22, \mathrm{p}=0.25$. Likewise, there was a negative correlation between the cardiologist's experience and fluoroscopy time $\mathrm{r}(28)=-0.32, \mathrm{p}=0.09$. After the exclusion of 3 significantly longer LAAO procedure times (in patients requiring a second occluder device) from the correlation analysis, we found a statistically significant $(<0.05,2$-tailed $)$ negative correlation between the cardiologist's experience and the procedural time $\mathrm{r}(25)=-0.43, \mathrm{p}=0.03$ and the fluoroscopic time $\mathrm{r}(25)=-0.40, \mathrm{p}=0.04$ (Fig. 3).

\section{Occluder size selection}

When selecting the occluder size, we always relied on the largest measured dimensions of the landing zone according to TEE and fluoroscopy. Figure 4 provides a comparison of the landing zone measurements with TEE and RTG and the final selected occluder device size.

The most frequently utilized occluder sizes were $28 \mathrm{~mm}$ (12 patients) and $25 \mathrm{~mm}$ (10 patients), while the least frequently utilized occluder sizes were $20 \mathrm{~mm}$ and $22 \mathrm{~mm}$, both placed in one patient only (Tab. 2).

\section{Indication subgroup analysis}

The contraindication to long-term anticoagulation treatment is the principal indication criterion for the LAAO procedure and, together with high $\mathrm{CHA}_{2} \mathrm{DS}_{2}$-VASc, it constitutes the inclusion criteria for this study population. The four patient groups are summarized based on the type of contraindication to long-term oral anticoagulation therapy ("LAAO indication groups") in Table 3.

We analysed the relationship between the LAAO indication groups and the patients' $\mathrm{CHA}_{2} \mathrm{DS}_{2}$-VASc and HASBLED scores using a one-way ANOVA and a significance level of $\mathrm{p}<0.05$ (Tab. 4).

We identified a statistically significant difference among the four indication groups in terms of the $\mathrm{CHA}_{2} \mathrm{DS}_{2}$-VASc score, $\mathrm{F}$ 
Tab. 3. Left Atrial Appendage Occlusion indication groups based on contraindications to long-term oral anticoagulation therapy

\begin{tabular}{lc}
\hline LAAO indication group & $\mathrm{n} \mathrm{( \% )}$ \\
\hline 1. Haemorrhagic stroke and/or a medical history of severe bleeding & $\mathbf{8 ~ ( 2 6 . 7 )}$ \\
Haemorrhagic stroke & 5 \\
Gastrointestinal bleeding with the need for transfusion & 2 \\
Bleeding in the eye followed by blindness & 1 \\
\hline 2. Medical history of recurrent or minor bleeding & $\mathbf{1 2}(\mathbf{4 0 . 0 )}$ \\
Gastrointestinal bleeding & 3 \\
Haematuria & 2 \\
Epistaxis & 2 \\
Recurrent non-specific bleeding & 2 \\
Spontaneous hematomas & 1 \\
Haemorrhoids & 1 \\
Morbus Crohn & 1 \\
\hline 3. Ischemic stroke despite adequate oral anticoagulation therapy & $\mathbf{3}(\mathbf{1 0 . 0 )}$ \\
\hline 4. Other reasons with high risk of bleeding & $\mathbf{7 ( 2 3 . 3 )}$ \\
Dialysis & 2 \\
Brain tumour & 1 \\
Intracranial aneurysm & 2 \\
Liver cirrhosis & 1 \\
Amyloid angiopathy & 1 \\
\hline
\end{tabular}

LAAO - Left Atrial Appendage Occlusion, 1After excluding other possible causes of stroke
$(3,26)=3.21, \mathrm{p}=0.039$. Indication group $1($ mean $=4.63, \mathrm{SD}=0.92)$ and indication group $3($ mean $=4.67, \mathrm{SD}=1.53)$ had higher $\mathrm{CHA}_{2} \mathrm{DS}_{2}$-VASc scores compared to indication group $2($ mean $=3.76, \mathrm{SD}=$ $1.37)$ and indication group $4($ mean $=2.86$; $\mathrm{SD}=1.07$ ), although post hoc testing with Gabriel's test failed to confirm that these differences were statistically significant.

There was a statistically significant difference among the four indication groups in terms of the HASBLED score, F $(3,26)$ $=5.39, \mathrm{p}=0.005$. Also, post hoc testing with Gabriel's test revealed significant differences between indication group 1 (M $=3.75, \mathrm{SD}=0.463)$ and indication group $4(\mathrm{M}=2.14, \mathrm{SD}=1.069)$. These findings indicate that patients in indication group 4 had lower HASBLED scores than patients in indication group 1.

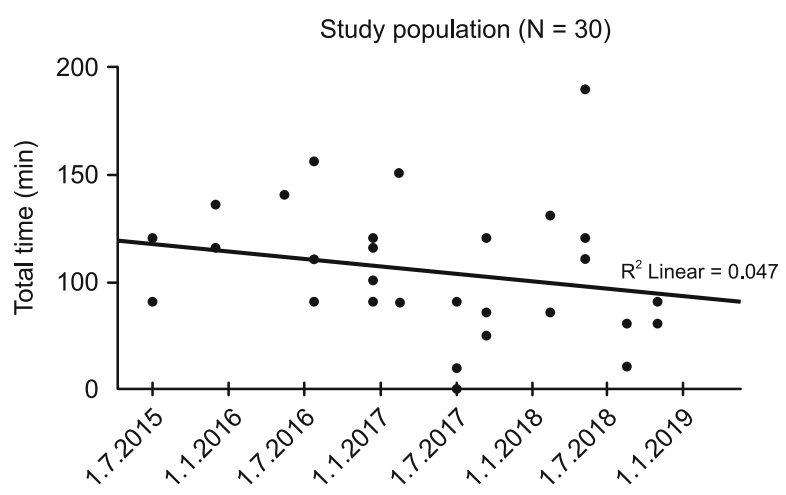

Date of procedure

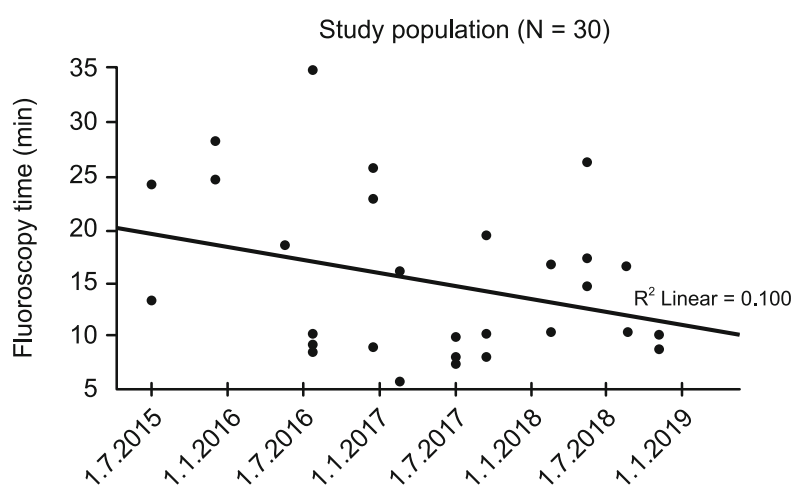

Date of procedure

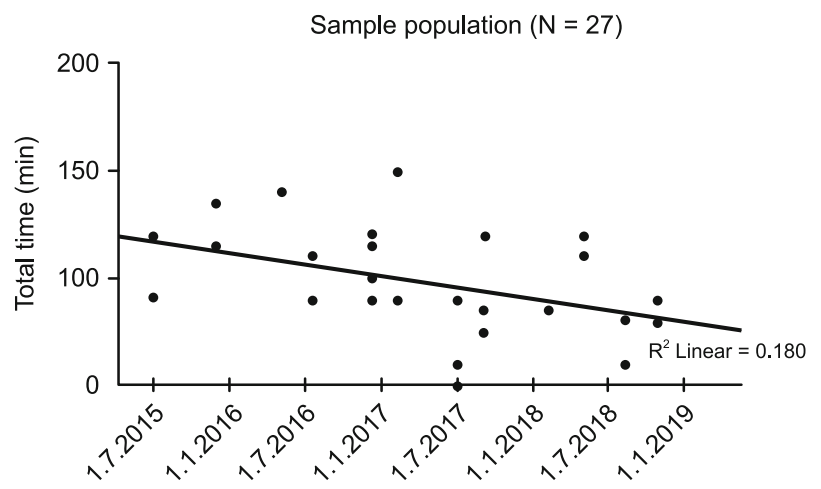

Date of procedure

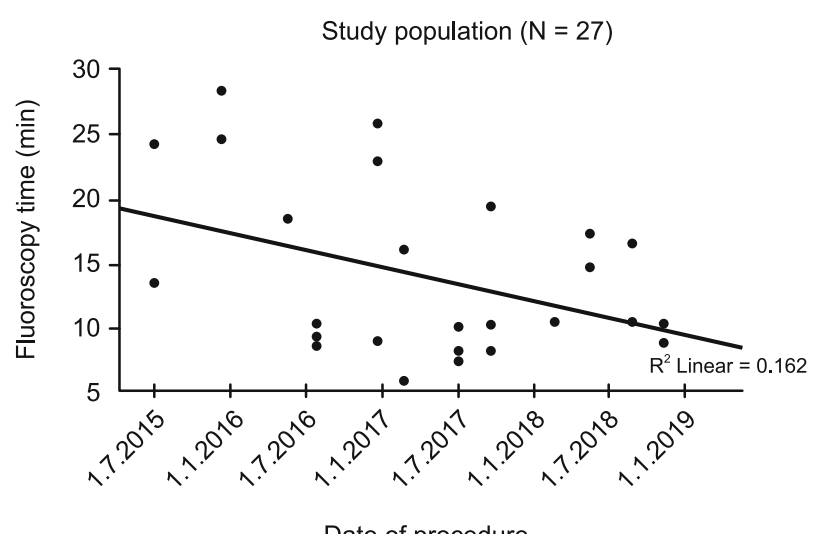

Date of procedure

Fig. 3. Relationship between cardiologist's experience and total procedure and fluoroscopy time. 
$609-618$

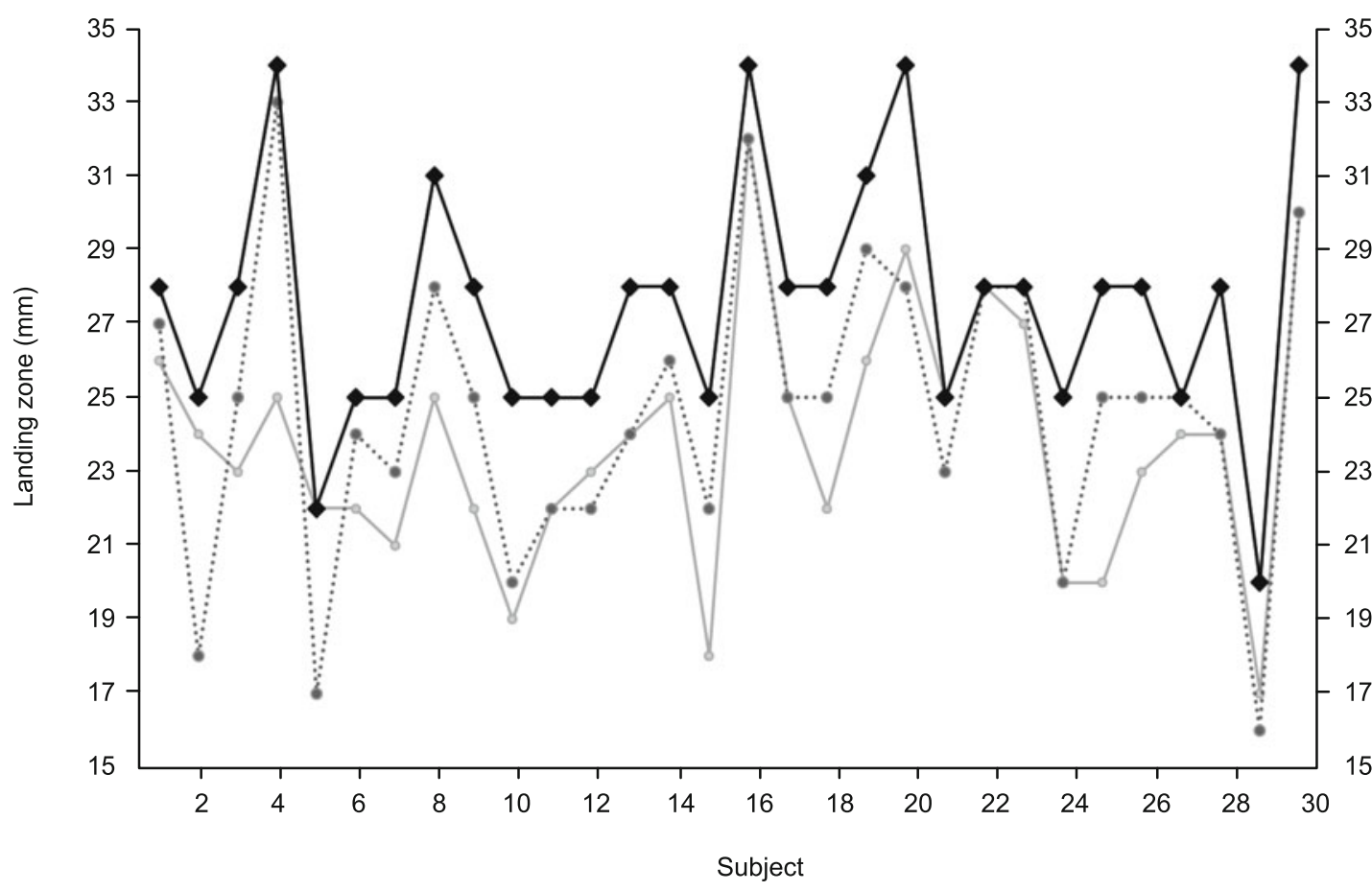

$\multimap$ Landing zone: TEE (mm) …. Landing zone: fluoroscopy $(\mathrm{mm}) \quad \leftrightarrow$ Occluder size

Fig. 4. Comparison of landing zone measurements with TEE and fluoroscopy regarding occluder size selection.

Follow-up after procedure

At the time of writing of this analysis, patients $(n=21)$ were monitored during regular follow-up visits for an average of approximately 23 months. All except the patient with the unsuccessful LAAO procedure attended the 1st follow up visit scheduled 3 months after LAAO, during which, all but one patient underwent TEE evaluation. The patient, who was not assessed with TEE (due to intolerance) was assessed by clinical anamnesis.

Twenty-one patients (70.0 \%) attended the 2 nd follow up visit, scheduled approximately 12 months after LAAO. Two patients died before the 2 nd follow up visit (causes of death were not related to the LAAO procedure) and six patients have not yet had the 2nd follow up visit.

Device thrombus formation has not been found in any of the patients. Furthermore, we observed no complications associated with interference of the occluder device in the mitral orifice or the upper pulmonary vein in any of our patients. We identified a hemodynamically minor leak in 4 patients $(1 \mathrm{~mm}$ and $2 \mathrm{~mm}$ leaks in 3 patients and 1 patient, respectively) (Tab. 5).

Tab. 4. Differences between indication groups at baseline.

\begin{tabular}{lccccccc}
\hline & $\begin{array}{c}\text { Group 1 } \\
(\mathrm{n}=8)\end{array}$ & $\begin{array}{c}\text { Group 2 } \\
(\mathrm{n}=12)\end{array}$ & $\begin{array}{c}\text { Group 3 } \\
(\mathrm{n}=3)\end{array}$ & $\begin{array}{c}\text { Group 4 } \\
(\mathrm{n}=7)\end{array}$ & $\begin{array}{c}\text { Total } \\
(\mathrm{n}=30)\end{array}$ & $\mathrm{F}$ & $\mathrm{p}$ \\
\hline CHA2DS2-VASc score & 4.63 & 3.67 & 4.67 & 2.86 & 3.83 & 3.21 & 0.029 \\
HASBLED score & 3.75 & 3.33 & 2.67 & 2.14 & 3.1 & 5.40 & 0.005 \\
\hline
\end{tabular}

$\mathrm{F}=\mathrm{F}$ statistic for a one-way ANOVA; $\mathrm{p}=$ level of significance
Further follow-up visits were scheduled on a yearly basis unless the patient's health condition warranted more frequent follow-up visits. No cases of cardioembolic stroke or peripheral embolization were observed in any of the patients during the analysed period.

\section{Pharmacotherapy}

Prior to LAAO, patients were treated mostly with low molecular weight heparin (LWMH) (16, 53.3\%) as shown in Table 6. Nine patients, 3 of whom had overcome an ischemic stroke despite oral anticoagulation therapy, $(30.0 \%)$ received DOAC. Four patients $(13.3 \%)$ used anti-aggregation mono-therapy or dual therapy. One patient $(3.3 \%)$ with a history of severe post-haemorrhagic anaemia in gastroduodenal bleeding received no antithrombotic treatment. None of the patients were treated with warfarin.

In the 3 months following the LAAO procedure, the occluder position was satisfactory and no hemodynamic leaks were observed; the majority of patients could continue with acetylsalicylic acid (ASA) or clopidogrel monotherapy, as summarized in Table 6.

One patient, in whom LAAO was unsuccessful, continued with LWMH. Two patients with relapsing ischemic stroke despite anticoagulation treatment continued with DOAC therapy. One patient with a relapsing ischemic stroke and haemorrhagic infarction continued to be treated with ASA. 
Tab. 5. Follow-up patient monitoring overview after Left Atrial Appendage Procedure.

\begin{tabular}{|c|c|}
\hline Sample & size $(n=30)$ \\
\hline \multicolumn{2}{|l|}{ 1st Follow-up Visit, n (\%) ${ }^{1}$} \\
\hline Patients attending & $29(96.7)$ \\
\hline Complications & $0(0)$ \\
\hline TEE Leak Evaluation & $28(96.6)$ \\
\hline Without leak & $24(85.7)$ \\
\hline $1 \mathrm{~mm}$ leak & $3(10.7)$ \\
\hline $2 \mathrm{~mm}$ leak & $1(3.6)$ \\
\hline TEE not evaluated & $1(3.4)$ \\
\hline Patients not attending & $1(3.3)$ \\
\hline Due to unsuccessful LAAO procedure & $1(100)$ \\
\hline \multicolumn{2}{|l|}{ 2nd Follow-up Visit, n (\%) ${ }^{1}$} \\
\hline Patients attending & $21(70)$ \\
\hline Complications & $0(0)$ \\
\hline TEE Leak Evaluation ${ }^{2}$ & $1(4.8)$ \\
\hline Without leak & $1(100)$ \\
\hline Patients not attending & $9(30)$ \\
\hline Due to unsuccessful LAAO procedure & $1(11.1)$ \\
\hline Due to exitus (cause of death: oncological disease) & $1(11.1)$ \\
\hline Due to exitus (cause of death under investigation) & $1(11.1)$ \\
\hline 2nd Follow-up Visit not performed by the time of analysis & $6(66.7)$ \\
\hline
\end{tabular}

Tab. 6. Antithrombotic medication.

\begin{tabular}{lccc}
\hline \multirow{2}{*}{ Antithrombotic medication } & $\begin{array}{c}\text { Baseline } \\
(\mathrm{n}=30)\end{array}$ & $\begin{array}{c}\text { After Month } 3 \\
(\mathrm{n}=28)\end{array}$ & $\Delta \%$ \\
\cline { 2 - 4 } & \multicolumn{3}{|c}{$\mathrm{n}(\%)$} \\
\hline ASA & $3(10.0)$ & $20(71.4)$ & +61.4 \\
Clopidogrel & $2(6.7)$ & $9(32.1)$ & +28.8 \\
LMWH & $16(53.3)$ & $0(0)$ & -53.3 \\
DOAC & $9(30.0)$ & $2(7.1)$ & -22.9 \\
No Treatment & $1(3.3)$ & $0(0.0)$ & -3.3 \\
\hline Detailed therapy & \multicolumn{3}{c}{} \\
\hline ASA & $2(6.7)$ & $17(60.7)$ & +54.0 \\
ASA and clopidogrel & $1(3.3)$ & $3(10.7)$ & +7.4 \\
Clopidogrel & $1(3.3)$ & $6(21.4)$ & +18.1 \\
LMWH & $16(53.3)$ & $0(0)$ & -53.3 \\
DOAC & $9(30.0)$ & $2(7.1)$ & -22.9 \\
No Treatment & $1(3.3)$ & $0(0)$ & -3.3 \\
\hline
\end{tabular}

ASA - acetylsalicylic acid, LMWH - low molecular weight heparin, DOAC - direct oral anticoagulants

\section{Discussion}

This study features our initial experience with the LAAO procedure, the successful LAA closure rate, and the benefits of this treatment during follow-up.

We were able to close the LAA in 28 of 30 patients $(93.3 \%)$ during the first procedure. In one patient, we discontinued the LAAO procedure due to thrombi formation during procedure. We assume that an insufficient heparin dose (Activated clotting time: 200-240 s) caused the thrombi formation. We removed the occluder and thrombi without complications. The second procedure on this patient was performed with high heparin doses (Activated clotting time: $300-350 \mathrm{~s}$ ) and it was without complications. Thus, the overall success rate of LAA closure in our patient population increased to $96.7 \%(29 / 30)$.

We failed to close the LAA in one patient with a chicken wing LAA. Despite the use of two occluder sizes ( 25 and $28 \mathrm{~mm}$ ), the position was deemed unstable with a high risk of later dislocation. We identified two main reasons that prevented successful LAA closure in this patient. First, the patient presented with a higher complexity in LAA anatomy and the cardiologist lacked the experience with the alternative sandwich method of LAA closure at the time of the procedure. Second, ICE was used instead of TEE because it was impossible to introduce the TEE probe in this patient. Although, we have several years of experience with ICE, especially in transseptal punctures, the different method of LAA imagining allowing only one projection might have contributed to procedure failure.

The overall success rate in our patient population was only slightly lower than the $97.3 \%$ success rate reported by Tsikas et al (6). On the other hand, we had only one minor periprocedural complication ( $3 \%$ incidence rate): the already described occluder thrombus. None of the patients experienced procedure-related pericardial effusion, stroke, air embolization or peripheral complications.

This study reports the results from an initial patient population undergoing LAAO at our institute. The first five patients underwent LAAO under the supervision of a proctor. With growing experience, we recorded shorter procedural and fluoroscopy times. Disregarding three patients in whom successful LAA was achieved with the second occluder size, the shortening of procedural times $(\mathrm{r}(25)=-0.43, \mathrm{p}=0.03)$ and fluoroscopic times $(\mathrm{r}(25)=-0.40 \mathrm{p}$ $=0.04)$ reached a statistical significance. For comparison, Masoud et al, reached the median procedural time of 81 minutes in their study of 83 patients (7).

According to professional literature, increasing cardiologist experience directly translates into a procedural success. Recent studies report high LAAO success rates (95-100\%) and decreased periprocedural complications (3-5\%) (8-11). Likewise, a large prospective multicentre registry of 1088 patients reported a successful LAA closure and periprocedural complications rates of $99 \%$ and $3.2 \%$, respectively (12).

We were successful in selecting the correct occluder size in 27 patients $(90 \%)$. Due to the initial miscalculation of the occluder size for three patients $(10 \%)$ a second size had to be used. Precise occluder size estimation requires an experience and cooperation with a trained echocardiographer. Therefore, the percentage of exact initial occluder size estimation varies significantly between the studies, described from $79.6 \%$ to $96.2 \%(13,14)$ Tsikas et al initially selected the correct occluder size in $93.3 \%$ of patients (6). Taking into consideration the fact that this is our initial patient population for LAAO at our institute, we consider the initial occluder size estimation as satisfactory.

In total, 28 of 29 patients $(97.0 \%)$ with a successful LAAO underwent a three-month TEE follow-up examination. TEE was not possible for one patient due to intolerance. The TEE followup rate in our patient population was very high compared to other studies. Tsikas et al reported that only $63.0 \%$ of patients under- 
$609-618$

went TEE follow-up (6). Regueiro et al reported TEE follow-up in $78.2 \%$ of patients (15).

Device-related thrombi and peri-device leaks during TEE examinations are considered potentially dangerous. A device leak may cause turbulent blood flow near the occluder that may increase platelet adherence resulting in thrombi formation at the occluder or in the residual part of the LAA. A small peri-device leak is not able to release a thrombus, thus leading to a low risk of systemic embolism. On the other hand, a large peri-device leak may lead to thrombus rupture with subsequent embolization (16). This may explain the conclusions of several studies where the presence of device-related thrombi and small leaks did not lead to increased thromboembolic complications (17). Tsikas et al documented leaks in $11.6 \%$ of their patients. Except for one patient, the presence of leaks did not require a re-initiation of anticoagulation therapy. On the other hand, the presence of device-related thrombi in $4.4 \%$ of the patients, required anticoagulation treatment, but without increasing thromboembolic complications (6). However in general, the occurrence of device related thrombus is considered an independent predictor of stroke and/or transient ischemic attack and thus should be managed accordingly with anticoagulation therapy (18). In our patient population, no device thrombi or hemodynamically severe leaks were detected (4 patients had a small leak of up to $2 \mathrm{~mm}$ ).

As of the last date of data collection, we had followed the patients on average for approximately 23 months, which corresponds with similar studies reporting a follow-up period from 4.5 to 30 months $(8,19)$. During this period, we observed no strokes, transient ischemic attacks, peripheral embolization or bleeding complications. Regueiro et al followed their study population of polymorbid patients with high $\mathrm{CHA}_{2} \mathrm{DS}_{2}$-VASc (mean: 4.8) and HASBLED (mean: 4.2) scores on average for four years. Their long-term follow-up confirmed the results from previous studies. The annual incidence of thromboembolic complications was $1.7 \%$, representing a $73 \%$ reduction in the relative risk estimated from the $\mathrm{CHA}_{2} \mathrm{DS}_{2}$-VASc score. Likewise, the annual incidence of bleeding complications was $4.8 \%$, representing a $47 \%$ reduction in the relative risk estimated from the HASBLED risk score (15).

The current AFib management guidelines issued by the European Society of Cardiology from 2016 categorize LAAO in indication group IIb. The essential indication criterion for LAAO is the contraindication of any anticoagulation therapy in patients at a high risk of AFib-related thromboembolism (1). The recommendations are based on the results of randomized clinical trials and registries $(6,20-23)$. However, majority of published randomized clinical trials include contraindication to long-term anticoagulation therapy among their exclusion criteria. Consequently, patients primarily undergoing LAAO in clinical practice are excluded from clinical trials.

Patients' risk profiles were assessed using $\mathrm{CHA}_{2} \mathrm{DS}_{2}$ VASc and HASBLED scores. Although, in clinical practice, we often encounter polymorbid patients, whose diseases and their combination are not considered within these scoring systems. Hence, the question of whether these patients meet LAAO indication criteria emerges. Since LAAO is an invasive treatment associated with a complication risk, we consider the correct and adequate indication as cru- cial. Therefore, as the health care centre with emerging LAAO experience, we carefully evaluated the risk-benefit profile of each patient eligible for LAAO. According to the 2019 expert consensus from the European Heart Rhythm Association and the European Association of Percutaneous Cardiovascular Interventions, LAAO may be considered for AFib patients at risk of ischemic stroke with an unacceptably high bleeding risk, refusing anticoagulant therapy or with a contraindication for systemic anticoagulation (24).

Based on the indications for LAAO, we divided our patient population into four indication groups. Patients in the first and second indication groups had a history of severe, life-threatening bleeding, haemorrhagic stroke or recurrent bleeding. Any anticoagulation therapy is absolutely contraindicated in these patients.

Patients in the third indication group experienced ischemic strokes despite an adequate anticoagulation therapy. Since further examinations excluded other causes, we assumed that the stroke was of cardioembolic origin; therefore LAAO was indicated for secondary stroke prevention in these patients. None of these patients experienced a stroke relapse in the assessed follow-up period. Alternatively, the 2016 Guidelines from the European Society of Cardiology on AFib management, recommend the following therapeutic approach for AFib patients with cardioembolic stroke despite an adequate anticoagulation therapy: increasing warfarin dose to reach an international normalized ratio of 2.5-3.5 or anticoagulation switch to DOAC, adding anti-aggregation treatment, if necessary. However, to date, there is no data to confirm the effectiveness of this approach (1). Hence, LAAO may be a suitable treatment alternative for these patients, as shown in the current study (24).

The fourth indication group included patients with heterogeneous reasons for LAAO indication. Although these patients are most prevalent in clinical practice, the HASBLED scores fail to identify their bleeding risk during anticoagulation treatment. Therefore, the mean HASBLED scores were significantly different in the four indication groups: $\mathrm{F}(3,26)=5.39, \mathrm{p}=0.005$ with the lowest mean HASBLED scores observed in the fourth indication group $(\mathrm{M}=2.14, \mathrm{SD}=1.069)$. They are frequently AFib patients with renal insufficiency for whom anticoagulation treatment is challenging due to regular dialysis. Recent data showed that LAAO is equally effective in dialyzed patients. Kefer et al reported that the use of ACP in LAAO for patients with renal insufficiency was equally safe, efficient, successful and equally reduced the risk of stroke and bleeding compared to the general population (25). In our patient population, we included five patients with renal insufficiency, of which two were dialyzed. Neither the LAAO procedure nor the follow-up results differed in these patients.

Furthermore, there are also diagnoses where anticoagulation therapy is not completely contraindicated, but significantly risky. Information on LAAO in these patients is limited in the current scientific literature for two main reasons: 1) difficult bleeding risk estimation; 2) low overall prevalence. We reported four patients in our study: one with amyloid angiopathy, one with a brain tumour at a high risk of bleeding and two with intracranial aneurysms. We observed no thromboembolic events in these patients during follow-up. 
It is crucially important to assess the risk-benefit ratio of LAAO in each patient individually, while considering that anti-aggregation therapy introduces a certain level of bleeding risk as well.

To date, there are no uniform guidelines for anti-aggregation and anticoagulation therapy after LAAO. The character and length of subsequent antithrombotic pharmacotherapy are based on the results of clinical studies and vary for different occluder types.

After LAAO with ACP Amulet ${ }^{\mathrm{TM}}$, antiaggregation therapy may be reduced to monotherapy during the first 3 months in patients at risk of high bleeding. In patients with an extremely high bleeding risk, no antiaggregation therapy may be considered (24). In our study, 23 patients $(76.7 \%$ ) received dual anti-aggregation with subsequent long-term anti-aggregation monotherapy. Pharmacotherapy varied among the remaining patients depending on their LAAO indication and concomitant diseases. In the Landmesser registry, $54 \%$ of the patients received dual anti-aggregation; $23 \%$ received anti-aggregation monotherapy, $18.9 \%$ remained on anticoagulation therapy, and $2 \%$ were discharged from the hospital without any treatment (12).

LAAO is an innovative method; however, since it has not been extensively tested, certain questions require attention.

To date, only two randomized clinical studies compared the safety and efficacy of LAAO (using Watchman device) with warfarin anticoagulant treatment (PROTECT AF and PREVAIL). However, the main limitation of these studies was the exclusion of patients with contraindications to anticoagulation treatment, who are highly prevalent in daily clinical practice $(20,21)$. Only registries and retrospective studies evaluated other types of occluders. Initial results from the PRAGUE-17 study comparing the safety, efficacy and effectiveness of LAAO with DOAC therapy the confirmed non-inferiority of LAAO (26) .

From an economic perspective, we stress that LAAO appears to be more cost-effective than life-long coagulation treatment associated with significant health complications, despite the higher initial costs (27-29). However, the economic value of LAAO will manifest only in patients with good health status assuring longterm survival (6).

The main limitation of the presented study is the small sample size. Our initial experience with the LAAO procedure, and as such our patient population, was limited to 30 patients, which includes all patients eligible for LAAO, who underwent LAAO at our institute in the last three and a half years.

Due to our limited experience with the LAAO procedure and potential serious complications, we very strictly considered the indication of LAAO in each patient. On the other hand, the completeness of data and high TEE follow-up rate need to be stressed.

Another limitation is the use of only one type of occluder device, since at the time of study initiation (year 2015), only one device, ACP Amulet was available at all clinics in Slovakia Therefore, the evaluation of LAAO with other devices and eventual comparison was not possible.

Selection bias might be present due to the observational retrospective design of the study. Data in the current study originated only from one health care centre and the performance of LAAO procedures, which may bias the presented results.
The current cohort non-randomized study investigated the patient characteristics and the effectiveness and safety of the study population undergoing LAAO; however, since it did not compare the LAAO procedure to any other therapeutic alternatives (other devices, procedures or pharmacotherapy), it had no control feature.

The average follow-up period covered by this analysis, considering the small sample size, does not provide a sufficient justification to draw definitive conclusions about the efficacy and safety of the LAAO procedure with the ACP Amulet ${ }^{\mathrm{TM}}$ device. Hence, these results need to be considered with caution.

The current study population is being closely monitored by the study team, while new patients are being considered for another larger study.

\section{Conclusions}

Based on the available data, LAAO appears to be an adequate alternative to anticoagulation therapy in patients with AFib at a high risk of stroke for whom anticoagulation therapy is contraindicated or associated with a high bleeding risk. The results of our study proved that LAAO was an effective and safe therapeutic approach, when the indication criteria and procedural steps are followed, even when performed at the health centre with a limited experience. It is to be assumed that ongoing studies will provide answers regarding the long-term effectiveness of the LAAO procedure and a comparison of different occluder types; they should also specify the type and length of antithrombotic therapy after the LAAO procedure; and specify the indication of LAAO in less frequently occurring situations.

\section{References}

1. Kirchhof P, Benussi S, Kotecha D, Ahlsson A, Atar D, Casadei B et al. ESC Guidelines for the management of atrial fibrillation developed in collaboration with EACTS. Eur Heart J 2016; 37 (38): 2893-962.

2. Savelieva I, Camm J. Update on Atrial Fibrillation: Part I. Clin Cardiol 2008 2008/02/01; 31 (2): 55-62.

3. Apostolakis S, Lane DA, Guo Y, Buller H, Lip GYH. Performance of the HEMORR2HAGES, ATRIA, and HAS-BLED Bleeding Risk-Prediction Scores in Patients With Atrial Fibrillation Undergoing Anticoagulation: The AMADEUS (Evaluating the Use of SR34006 Compared to Warfarin or Acenocoumarol in Patients With Atrial Fibrillation) Study. J Amer Coll Cardiol 2012; 60 (9): 861-867.

4. Blackshear JL, Odell JA. Appendage obliteration to reduce stroke in cardiac surgical patients with atrial fibrillation. Ann Thorac Surg 1996; 61 (2): 755-759.

5. Sievert H, Lesh Michael D, Trepels T, Omran H, Bartorelli A, Della Bella P et al. Percutaneous Left Atrial Appendage Transcatheter Occlusion to Prevent Stroke in High-Risk Patients With Atrial Fibrillation. Circulation 2002; 105 (16): 1887-1889.

6. Tzikas A, Shakir S, Gafoor S, Omran H, Berti S, Santoro G et al. Left atrial appendage occlusion for stroke prevention in atrial fibrillation: multicentre experience with the AMPLATZER Cardiac Plug. EuroIntervention 2016; 11 (10): 1170-1179. 
$609-618$

7. Masoud A, Bartoletti S, Fairbairn T, Khurana A, Velavan P, Morrison WL et al. Outcome of left atrial appendage occlusion in high-risk patients. Heart 2018; 104 (7): 594.

8. Berti S, Pastormerlo LE, Rezzaghi M, Trianni G, Paradossi U, Cerone $\mathbf{E}$ et al. Left atrial appendage occlusion in high-risk patients with non-valvular atrial fibrillation. Heart 2016; 102 (24): 1969-1973.

9. Santoro G, Meucci F, Stolcova M, Rezzaghi M, Mori F, Palmieri C et al. Percutaneous left atrial appendage occlusion in patients with nonvalvular atrial fibrillation: implantation and up to four years follow-up of the AMPLATZER Cardiac Plug. EuroIntervention 2016; 11 (10): 1188-1194.

10. Kleinecke C, Park JW, Gödde M, Zintl K, Schnupp S, Brachmann J. Twelve-month follow-up of left atrial appendage occlusion with Amplatzer Amulet. Cardiol J 2017; 24 (2): 131-138.

11. Nielsen-Kudsk JE, Johnsen SP, Wester P, Damgaard D, Airaksinen $\mathbf{J}$, Lund $\mathbf{J}$ et al. Left atrial appendage occlusion versus standard medical care in patients with atrial fibrillation and intracerebral haemorrhage: a propensity score-matched follow-up study. EuroIntervention : journal of EuroPCR in collaboration with the Working Group on Interventional Cardiology of the European Society of Cardiology 2017; 13 (3): 371-378.

12. Landmesser U, Schmidt B, Nielsen-Kudsk JE, Lam SCC, Park JW, Tarantini G et al. Left atrial appendage occlusion with the AMPLATZER Amulet device: periprocedural and early clinical/echocardiographic data from a global prospective observational study. EuroIntervention 2017; 13 (7): 867-876.

13. López-Mínguez JR, González-Fernández R, Fernández-Vegas C, Millán-Nuñez V, Fuentes-Cañamero ME, Nogales-Asensio JM et al. Anatomical Classification of Left Atrial Appendages in Specimens Applicable to CT Imaging Techniques for Implantation of Amplatzer Cardiac Plug. Journal of Cardiovascular Electrophysiology 2014; 25 (9): 976-984.

14. Park JW, Bethencourt A, Sievert H, Santoro G, Meier B, Walsh K et al. Left atrial appendage closure with Amplatzer cardiac plug in atrial fibrillation: initial European experience. Catheter Cardiovasc Interv 2011; 77 (5): 700-706.

15. Regueiro A, Cruz-Gonzalez I, Bethencourt A, Nombela-Franco L, Champagne J, Asmarats L et al. Long-term outcomes following percutaneous left atrial appendage closure in patients with atrial fibrillation and contraindications to anticoagulation. J Intervent Cardiac Electrophysiol 2018; 52 (1): 53-59.

16. Viles-Gonzalez JF, Kar S, Douglas P, Dukkipati S, Feldman T, Horton R et al. The Clinical Impact of Incomplete Left Atrial Appendage Closure with the Watchman Device in Patients with Atrial Fibrillation. A PROTECT AF (Percutaneous Closure of the Left Atrial Appendage Versus Warfarin Therapy for Prevention of Stroke in Patients with Atrial Fibrillation). Substudy 2012; 59 (10): 923-929.

17. Saw J, Tzikas A, Shakir S, Gafoor S, Omran H, Nielsen-Kudsk JE et al. Incidence and Clinical Impact of Device-Associated Thrombus and PeriDevice Leak Following Left Atrial Appendage Closure With the Amplatzer Cardiac Plug. JACC: Cardiovasc Intervent 2017; 10 (4): 391-399.
18. Fauchier L, Cinaud A, Brigadeau F, Lepillier A, Pierre B, Abbey S et al. Device-Related Thrombosis After Percutaneous Left Atrial Appendage Occlusion for Atrial Fibrillation. J Am Coll Cardiol 2018; 71 (14): 1528-3156.

19. Guerios EE, Schmid M, Gloekler S, Khattab AA, Wenaweser PM, Windecker $\mathrm{S}$ et al. Left atrial appendage closure with the Amplatzer cardiac plug in patients with atrial fibrillation. Arquivos Brasil Cardiol 2012; 98 (6): 528-356.

20. Holmes DR, Kar S, Price MJ, Whisenant B, Sievert H, Doshi SK et al. Prospective Randomized Evaluation of the Watchman Left Atrial Appendage Closure Device in Patients With Atrial Fibrillation Versus LongTerm Warfarin Therapy. J Amer Coll Cardiol 2014; 64 (1): 1.

21. Holmes DR, Reddy VY, Turi ZG, Doshi SK, Sievert H, Buchbinder M et al. Percutaneous closure of the left atrial appendage versus warfarin therapy for prevention of stroke in patients with atrial fibrillation: a randomised non-inferiority trial. Lancet 2009; 374 (9689): 534-542.

22. Sick PB, Schuler G, Hauptmann KE, Grube E, Yakubov S, Turi ZG et al. Initial Worldwide Experience With the WATCHMAN Left Atrial Appendage System for Stroke Prevention in Atrial Fibrillation. J Amer Coll Cardiol 2007; 49 (13): 1490-1495.

23. Boersma LV, Schmidt B, Betts TR, Sievert H, Tamburino C, Teiger E et al. Implant success and safety of left atrial appendage closure with the WATCHMAN device: peri-procedural outcomes from the EWOLUTION registry. Eur Heart J 2016; 37 (31): 2465-2474.

24. Glikson M, Wolff R, Hindricks G, Mandrola J, Camm AJ, Lip GYH et al. EHRA/EAPCI expert consensus statement on catheter-based left atrial appendage occlusion - an update. EP Europace 2019.

25. Kefer J, Tzikas A, Freixa X, Shakir S, Gafoor S, Nielsen-Kudsk JE et al. Impact of chronic kidney disease on left atrial appendage occlusion for stroke prevention in patients with atrial fibrillation. Internat J Cardiol 2016; 207: 335-340.

26. https://clinicaltrials.gov/ct2/show/NCT02426944? cond=PRAGUE+ $17 \&$ rank $=1$.

27. Amorosi SL, Armstrong S, Da Deppo L, Garfield S, Stein K. The budget impact of left atrial appendage closure compared with adjusted-dose warfarin and dabigatran etexilate for stroke prevention in atrial fibrillation. EP Europace 2014; 16 (8): 1131-1136.

28. Reddy VY, Akehurst RL, Armstrong SO, Amorosi SL, Brereton N, Hertz DS et al. Cost effectiveness of left atrial appendage closure with the Watchman device for atrial fibrillation patients with absolute contraindications to warfarin. EP Europace 2016; 18 (7): 979-986.

29. Reddy VY, Akehurst RL, Armstrong SO, Amorosi SL, Beard SM, Holmes DR, Jr. Time to Cost-Effectiveness Following Stroke Reduction Strategies in AF: Warfarin Versus NOACs Versus LAA Closure. J Am Coll Cardiol 2015; 66 (24): 2728-2739.

Received April 9, 2020. Accepted June 15, 2020. 